\title{
Expression and clinical significance of Beclin-1 in gastric cancer tissues of various clinical stages
}

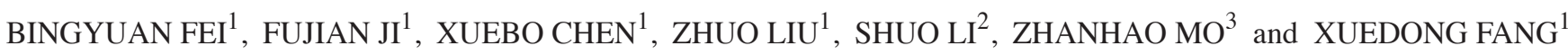 \\ Departments of ${ }^{1}$ General Surgery and ${ }^{2}$ Hepatobiliary and Pancreas Surgery; ${ }^{3}$ Endoscopy Center, \\ The China Japan Union Hospital of Jilin University, Changchun, Jilin, P.R. China
}

Received November 11, 2014; Accepted September 4, 2015

DOI: $10.3892 / \mathrm{ol} .2016 .4183$

\begin{abstract}
Autophagy is a common phenomenon in cancer metabolism. However the mechanism and guiding significance of autophagy in the development of gastric cancer has remained to be elucidated. In the present study, 75 gastric cancer tissue specimens were collected at The China Japan Union Hospital of Jilin University (Changchun, China). Of these samples, 16 cases were stage 1,40 stage 2 and 19 stage 3. Polymerase chain reaction and western blotting were used to detect the messenger RNA and protein expression of Beclin-1, a significant protein associated with cellular autophagy. It was found that expression of Beclin-1 in cancer tissues from stages 1 and 2 was higher, while in stage 3 cases levels were significantly lower than that of adjacent normal tissues. In addition, the infiltration of inflammatory cytokines was also increased in stage 1 and 2 cases. In vitro studies revealed that following stimulation with interferon- $\gamma$ (IFN- $\gamma$ ), autophagy-associated proteins Beclin-1 and microtubule-associated protein light chain 3 were activated. Furthermore, activation of autophagy inhibited xenograft growth in nude mice. The results of these in vivo and in vitro experiments indicated that in gastric cancer tissues, autophagy was downregulated following the development of cancer tissue and that inflammation may be a significant factor in this process. IFN- $\gamma$ may be involved in the mediation of this process and thus present a novel target for the treatment of gastric cancer.
\end{abstract}

\section{Introduction}

The morbidity of gastric cancer is particularly high in China, where $23.9 \%$ of the total death toll resulting from tumors was attributed to patients with gastric cancer in 2012 (1). Although favorable therapeutic outcomes for the treatment

Correspondence to: Professor Xuedong Fang, Department of General Surgery, The China Japan Union Hospital of Jilin University, 829 Xin'min Road, Changchun, Jilin 130012, P.R. China E-mail: fang_xuedongjl@163.com

Key words: autophagy, gastric, cancer, Beclin-1, interferon- $\gamma$ of gastric cancer have been achieved by a combination of surgery and chemotherapy, distant metastasis and recurrence remain common in advanced stage patients $(2,3)$. Progression of the tumor cells may be induced by a variety of factors, including multiple structural and functional genetic alterations, cell-cycle regulators, adhesion molecules and growth factors (2). Study of these factors is of significant clinical value for further understanding the underlying molecular mechanisms of gastric cancer progression, as well as identifying novel therapeutic targets in gastric tissues (4-6).

Autophagy is a common occurrence in cell metabolism, and is involved in facilitating the cellular clearance of aggregation-prone proteins, thus exerting a cytoprotective function (7). Autophagy is a genetically controlled process, including lysosomal degradation and recycling of these proteins and cellular organelles. The process of autophagy is activated under various conditions, including hypoxia, inflammatory stimulation and lack of nutrients (8). Activation of autophagy has two potential effects, depending on the environment; promoting non-apoptotic type II programmed cell death or facilitating adaptive cell survival (9-11). Cancer cells are able to survive under hypoxic and hypo-nutrient microenvironments, and therefore theoretically enhance autophagic ability to utilize recyclable materials. However, there is evidence that autophagy may be capable of promoting and suppressing cancer. Beclin-1, the first identified mammalian autophagy effector, was reported to be deleted or significantly decreased in ovarian, breast and prostate cancer, suggesting that autophagy may be a tumor-suppressive mechanism (12). Notably, previous studies have indicated that low expression levels of Beclin-1 were predictive of poor survival in B cell lymphoma-xL-overexpressing hepatocellular carcinoma tissues $(2,3)$. These observations indicate that autophagy has a significant role in tumorigenesis and prognosis. However, there have been limited studies regarding the roles of autophagy in gastric cancer specifically (13).

In the present study, the expression of autophagy-associated protein Beclin-1 and inflammatory cytokine interferon- $\gamma$ $($ IFN- $\gamma)$ were evaluated in clinical gastric cancer tissue samples and cell lines. The association between autophagy and inflammation was also examined. The present study aimed to elucidate the characteristics of autophagy in gastric cancer and the effects of inflammation on this process. 


\section{Materials and methods}

Patients and tissue samples. Between January 2010 and December 2014, clinical gastric cancer and adjacent noncancerous gastric mucosa tissue specimens were collected from 75 patients who had undergone surgeries for radical resection of gastric cancer at The China Japan Union Hospital of Jilin University (Changchun, China). The study protocol was approved by the institutional review board for human studies of The China Japan Union Hospital of Jilin University, and informed consent was obtained from all patients. Following collection, the tissues were divided into two parts, a large part $(50-100 \mathrm{mg})$ and a small one $(10 \mathrm{mg})$. The $50-100 \mathrm{mg}$ samples were immediately frozen and stored in liquid nitrogen for western blot analysis and quantitative polymerase chain reaction (qPCR), while the $10 \mathrm{mg}$ samples were washed with phosphate-buffered saline (PBS; Wuhan Boster Biological Technology, Ltd., Wuhan, China) and stored in 4\% paraformaldehyde (Wuhan Boster Biological Technology, Ltd.) for further histological analysis. Details of the patients are listed in Table I.

Cell culture and stimulation. Human gastric cancer cell lines BGC-823 and MKN-28 (Cell Bank of the Chinese Academy of Sciences, Shanghai, China), and human normal gastric mucosa epithelial cell line GES-1 (Cell Bank of the Chinese Academy of Sciences, Shanghai, China), were grown in Dulbecco's modified Eagle's medium supplemented with $10 \%$ fetal bovine serum (FBS; HyClone; GE Healthcare Life Sciences, Logan, UT, USA), $100 \mathrm{U} / \mathrm{ml}$ penicillin and $100 \mathrm{~g} / \mathrm{ml}$ streptomycin (Sangon Biotech, Shanghai, China). Cells were tested for authenticity by the Cell Bank of the Chinese Academy of Sciences prior to purchase. Cells were maintained at $37^{\circ} \mathrm{C}$ in a humidified atmosphere of $5 \% \mathrm{CO}_{2}$. Cells were plated in 6-well plates and experimental group cells were stimulated with $50 \mathrm{ng} / \mathrm{ml}$ IFN- $\gamma$ (BioLegend, Inc., San Diego, CA, USA) for 12, 24 and $48 \mathrm{~h}$ (14). The control group cells received the same dose of PBS. Expression levels of Beclin-1 and LC3 were analyzed by western blot analysis.

Animal experiments. Female BALB/c nude mice (18-20 g, 6 weeks of age) were purchased from Huafukang Co. Ltd. (Beijing, China). In total, 50 mice were used for the experiment, of which 10 were used for the tumor bearing assay and 40 were used for the xenograft assay, with 20 mice in the experimental group and 20 mice in the control group. The mice were bred at the animal center of Jilin University and were maintained according to the Jilin University animal management program. Mice were housed under specific pathogen-free conditions at a temperature of $23 \pm 1^{\circ} \mathrm{C}$ and relative humidity of $30-70 \%$. Mice were maintained in a 12-h light-dark cycle, with free access to water and ad libitum feeding with sterilized food. Exponentially growing gastric cancer cells (BGC-823 and MKN-28 cells; $5 \times 10^{6}$ cells in $200 \mu$ l PBS) were subcutaneously inoculated into the left hind leg of 10 mice. Tumor-bearing mice were sacrificed 10 days later by $\mathrm{CO}_{2}$ inhalation, and the tumors were dissected and divided into isometrical tissue blocks ( $2 \mathrm{~mm}$ in diameter). The tissue blocks were implanted into the back of 40 novel nude mice. These xenograft tumors were allowed to grow for a further 14 days. Then the mice
Table I. Details of patients included in the present study $(n=75)$.

\begin{tabular}{lc}
\hline Parameter & Patients, $\mathrm{n}$ \\
\hline Gender & \\
Male & 47 \\
Female & 28 \\
Median age, years (range) & $54(38-65)$ \\
Median BMI, kg $/ \mathrm{m}^{2}$ (range) & $24.3(17.6-28.4)$ \\
TNM stage & \\
I & 16 \\
II & 40 \\
III & 19 \\
\hline
\end{tabular}

BMI, body mass index; TNM, tumor/node/metastasis (13).

were randomly divided into two groups (20 mice per group). The control group received a subcutaneous injection of saline (50 $\mu$ 1; Qingdao Hope Bio-Technology Co., Ltd., Qingdao, China) every 2 days for 14 days, while the treatment group received a subcutaneous injection of IFN- $\gamma(0.5 \mathrm{ng}$ in $50 \mu \mathrm{l})$ every 2 days for 14 days. Tumor volume [(major axis x minor axis $)^{2} \times 0.5$ ] (15) and status of the mice were measured every day for 14 days from the commencement of treatment.

Immunohistochemistry. A total of 53 clinical gastric cancer and adjacent noncancerous gastric mucosa tissue specimenscarcinomas tissues previously fixed in paraformaldehyde were used for immunohistochemistry study here. These samples were triformol-fixed, dehydrated in a graded alcohol series, embedded in paraffin and cut into sections. The aforementioned chemical reagents were purchased from Sinopharm Chemical Reagent Co., Ltd. (Shanghai, China). The sections were subsequently deparaffinized, blocked with $3 \%$ hydrogen peroxide for $10 \mathrm{~min}$, washed with deionized water three times and sealed with serum (FBS; HyClone; GE Healthcare Life Sciences) for $30 \mathrm{~min}$ at room temperature. The sections were washed with deionized water and incubated for $30 \mathrm{~min}$ at $37^{\circ} \mathrm{C}$ with primary polyclonal rabbit anti-human Beclin-1 antibodies (1:500 in 1\% BSA in PBS containing 0.1\% Tween; cat no. ab62472; Abcam, Cambridge, MA, USA). Next, the sections were washed three times with PBS and incubated with a biotinylated goat anti-rabbit IgG secondary antibody (1:300 in $1 \%$ BSA in PBS containing $0.1 \%$ Tween; cat. no. ab64257; Abcam) for $20 \mathrm{~min}$ at room temperature. The sections were washed three times, followed by detection with the HRP Substrate Kit and ABC kit (SK-4100 and PK-6100, protocols provided in the kit. Vector Blue; Vector Laboratories Inc., Burlingame, CA, USA). and nuclei were counterstained with hematoxylin using the ULTRA Staining system (Ventana Medical Systems, Tucson, AZ, USA). Negative controls were obtained by substituting PBS for the primary antibody. The results were evaluated based on the proportion of stained cells and the staining intensity.

Tissues were interpreted as positive when a minimum of weak-to-moderate cytoplasmic staining was identified in $>30 \%$ of the neoplastic cells. The rates of Beclin-1-positive 
Table II. Beclin-1 expression in various types of gastric carcinoma.

\begin{tabular}{|c|c|c|c|c|}
\hline \multirow[b]{2}{*}{ Gastric carcinoma characteristic } & \multicolumn{4}{|c|}{ Beclin-1 expression, n (\%) } \\
\hline & - & + & ++ & Total positive \\
\hline \multicolumn{5}{|l|}{ Histological grade } \\
\hline Well & 3 & 9 & 11 & $20(86.96)$ \\
\hline Moderate & 7 & 12 & 18 & $30(81.10)$ \\
\hline Poor & 12 & 2 & 1 & $3(20.00)^{\mathrm{a}}$ \\
\hline \multicolumn{5}{|l|}{ Lymph node metastasis } \\
\hline No & 6 & 6 & 8 & $14(65.00)$ \\
\hline Yes & 15 & 17 & 22 & $39(70.90)$ \\
\hline \multicolumn{5}{|l|}{ Depth of invasion } \\
\hline EGC & 3 & 4 & 15 & $19(83.63)^{\mathrm{b}}$ \\
\hline AGC & 19 & 19 & 15 & $34(64.15)$ \\
\hline
\end{tabular}

EGC, early gastric cancer; AGC, advanced gastric cancer; -, 0-20\% cells Beclin- 1 positive; +, 30-60\% cells Beclin- 1 positive; ++, $\geq 61 \%$ cells Beclin-1 positive. ${ }^{\mathrm{a}} \mathrm{P}<0.01$ vs. well- and moderately-differentiated cancer; ${ }^{\mathrm{b}} \mathrm{P}<0.05$ vs. AGC.

cells were graded as ++ when $\geq 61 \%$ of the cells were positive, + when $30-60 \%$ were positive, and - when $0-29 \%$ were positive. Results were independently reviewed by two pathologists.

$q P C R$. RNA in the clinical tissue samples was isolated using mechanical homogenization (LabGEN 700 Homogenizer; Cole-Parmer, Vernon Hills, IL, USA) and TRIzol reagent (Invitrogen; Thermo Fisher Scientific, Inc., Waltham, MA, USA). Complementary DNA was synthesized from the total RNA using SuperScriptIII RNase H-Reverse Transcriptase (Invitrogen; Thermo Fisher Scientific, Inc.). The expression levels of the genes were quantified using TaqMan ${ }^{\circledR}$ Gene Expression assays (Applied Biosystems Life Technologies, Foster City, CA, USA). The results were expressed as relative expression, standardized against the expression of the gene encoding $\beta$-actin. $\beta$-actin was used as the cDNA loading control, and all qPCR read-outs were adjusted according to the $\beta$-actin results. The primers used were as follows: Beclin-1 forward, 5'-CAAGATCCTGGACCGTGTCA-3' and reverse, 5'-TGGCACTTTCTGTGGACATCA-3'; $\beta$-actin forward, 5'-TGGCACCCAGCACAATGAA-3' and reverse, 5'-CTAAGTCATAGTCCGCCTAGAAGCA-3' (16).

Western blot analysis. Cytoplasmic proteins were extracted from gastric cancer cell lines and clinical samples using NE-PER Nuclear and Cytoplasmic Extractions reagents (Beyotime Institute of Biotechnology, Haimen, China). The lysates were resolved by $4-20 \%$ SDS-PAGE gradient gels (Bio-Rad Laboratories, Inc., Hercules, CA, USA) and transferred onto polyvinylidene difluoride membranes (EMD Millipore, Billerica, MA, USA). Blots were blocked and incubated with rabbit anti-human polyclonal primary antibodies against Beclin-1 (cat no. ab62472), LC3 (cat no. ab128025) and GADPH (cat no. ab37168) at dilutions of 1:1,000 at $4^{\circ} \mathrm{C}$ overnight (Abcam). Blots were then incubated with a secondary goat anti-rabbit antibody (cat. no. sc-2004; 1:5,000 dilution; Santa Cruz Biotechnology, Inc., Dallas, TX, USA) at room temperature for $1 \mathrm{~h}$. Blots were visualized using enhanced chemiluminescence detection reagents and then exposed to X-ray film (Thermo Fisher Scientific, Inc.) (17,18).

Fluorescence-activated cell sorting (FACS) analysis for intracellular IFN- $\gamma$. FACS requires a small number of high quality tissue samples and is expensive to run, therefore, only 5 of the 75 fresh clinical samples were selected for analysis. The samples were divided into sections and ground on a screen cloth. PBS was used to wash the ground tissues, filtering immune cells through the cloth by the wash buffer (PBS) and separating them from the cell debris. These cells were collected by centrifugation of the washing liquid and stained with phycoerythrin-CD3, allophycocyanin-CD4 and fluorescein isothiocyanate-IFN- $\gamma$ monoclonal antibodies (BioLegend, Inc.). Data were analyzed using the BD FACS CantoII (BD Biosciences, Franklin Lakes, NJ, USA) (19).

Statistical analysis. Data are expressed as the mean \pm standard error of the mean. Statistical significance was determined by the Student's t-test, Mann-Whiney U test or Fisher's exact test. $\mathrm{P}<0.05$ was considered to indicate a statistically significant difference.

\section{Results}

Expression of Beclin-1 in gastric cancer tissues and cell lines. Immunohistochemistry and western blotting were used to analyze the expression levels of Beclin-1 in gastric cancer tissues and cell lines. As shown in Fig. 1, in tissues of tumor/node/metastasis (TNM) stage I and II, the expression of Beclin-1 was higher than that in the adjacent normal tissues $(\mathrm{P}<0.05)$. However, in tissues of TNM stage III, expression of Beclin-1 was reduced compared with that of the adjacent tissue $(\mathrm{P}<0.05)$. The results of immunohistochemistry and western blot analysis were concurrent.

The expression of Beclin-1 in BGC-823, MKN-28 and normal gastric mucosa epithelial cell line GES-1 were analyzed by western blotting. BGC-823 is a 


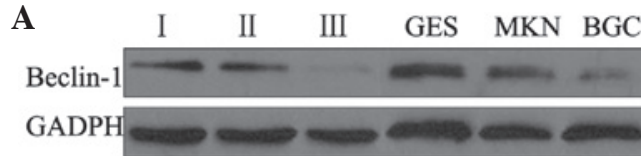

C

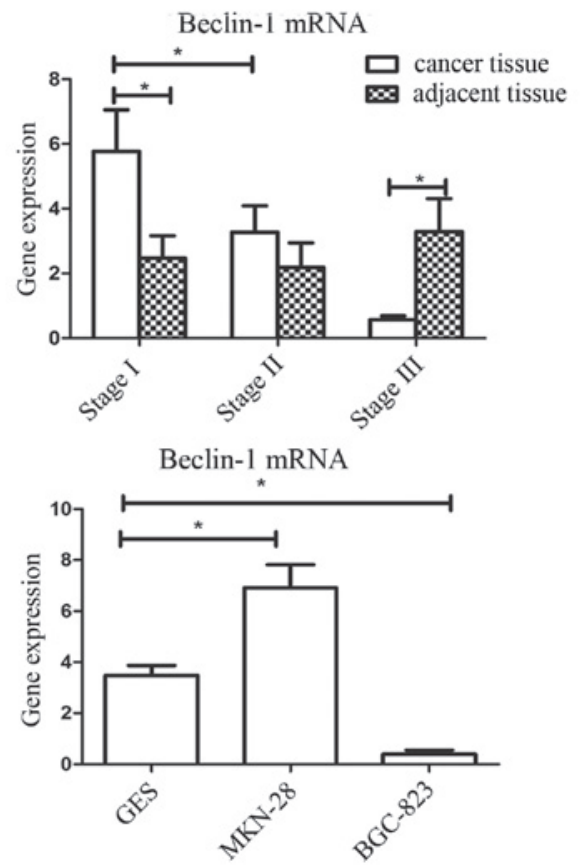

$\mathbf{B}$

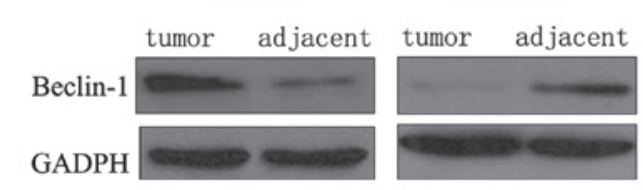

D

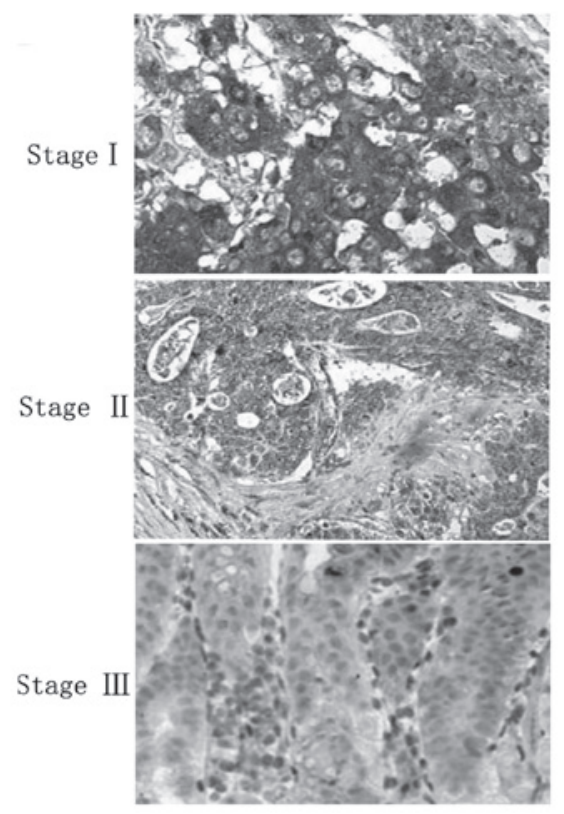

Figure 1. Expression of Beclin-1 in gastric cancer tissues and cell lines. (A) Western blot analysis of clinical samples of various stage gastric cancer and cell lines. (B) Expression levels of Beclin-1 in stage I and III cancer and adjacent tissues. (C) Expression of Beclin-1 mRNA in gastric cancer tissues and cells, evaluated by polymerase chain reaction. Values are expressed as the mean \pm standard error of the mean, $\mathrm{P}<0.05$. (D) Visualization of Beclin-1 expression in gastric cancer tissues of various stages by immunohistochemistry (magnification, x100). Bec-1, Beclin-1; mRNA, messenger RNA; GES, GES-1 cells; MKN, MKN-28 cells; BGC, BCG-823 cells.

poorly-differentiated gastric adenocarcinoma cell line, while MKN-28 is a well-differentiated adenocarcinoma cell line. It was observed that the expression of Beclin-1 in the poorly-differentiated BGC-823 cell line was significantly lower compared with that of the other cells $(\mathrm{P}<0.05)$. This result was in accordance with the results of the analysis of clinical tissue samples. However, significant differences in Beclin-1 expression were not observed between the well-differentiated MKN-28 and the normal GES-1 cell lines.

Beclin-1 expression is correlated with differentiation and depth of invasion. Immunopositivity for Beclin-1 was observed in 53/75 gastric adenocarcinoma patient samples analyzed in the present study. Expression of Beclin-1 was observed in $20.00 \%$ of the poorly-differentiated samples, $81.10 \%$ of the moderately-differentiated samples and $86.96 \%$ of the well-differentiated samples. There were significant differences between the poorly-differentiated group and the well- and moderately-differentiated groups $(\mathrm{P}<0.01$; two-tailed Fisher's exact test). There were no significant differences in Beclin-1 expression with respect to the existence of lymph node metastasis. However, the expression of Beclin-1 was associated with the depth of invasion. Samples of early gastric cancer expressed higher levels of Beclin-1 than those of advanced gastric cancer. Details are presented in Table II.
Infiltration of IFN- $\gamma$ in cancer tissues. FACS analysis was used to evaluate the percentage of immune cells secreting IFN- $\gamma$ (Fig. 2A and B). Five fresh samples of a suitable size were selected for FACS analysis. Western blotting was also used to directly study the protein expression of IFN- $\gamma$ (Fig. 2C). In the present study, it was demonstrated that the majority of immune cells which were IFN- $\gamma$ positive, were CD3(+)CD4(+), indicating that they were Th1 cells. However, the percentage of IFN- $\gamma$-positive cells in stage I/II cases was significantly higher than that of cases of stage III (Fig. 2).

IFN- $\gamma$ induces autophagy in gastric cancer cells. IFN- $\gamma$ was subsequently used to induce autophagy in gastric cancer cells. Beclin-1 was originally highly expressed in MKN-28 cells, while rarely expressed in BGC-823 cells. The poorly-differentiated BGC-823 cell line was therefore selected for this in vitro experiment. In order to investigate whether autophagy was induced in the BGC-823 cells, the expression levels of autophagy-associated proteins LC3 and Beclin-1 were analyzed. As shown in Figs. 1 and 3, in the control cells, low levels of LC3 and Beclin-1 were detected, however, following treatment with IFN- $\gamma$ for $48 \mathrm{~h}$, the expression levels of these two proteins increased significantly (Fig. 3A). Furthermore, the ratio of LC3-II/LC3-I (represented by the two bands) increased significantly compared with that of the control groups, indicating the activation of autophagy in these cells (Fig. 3). 
A

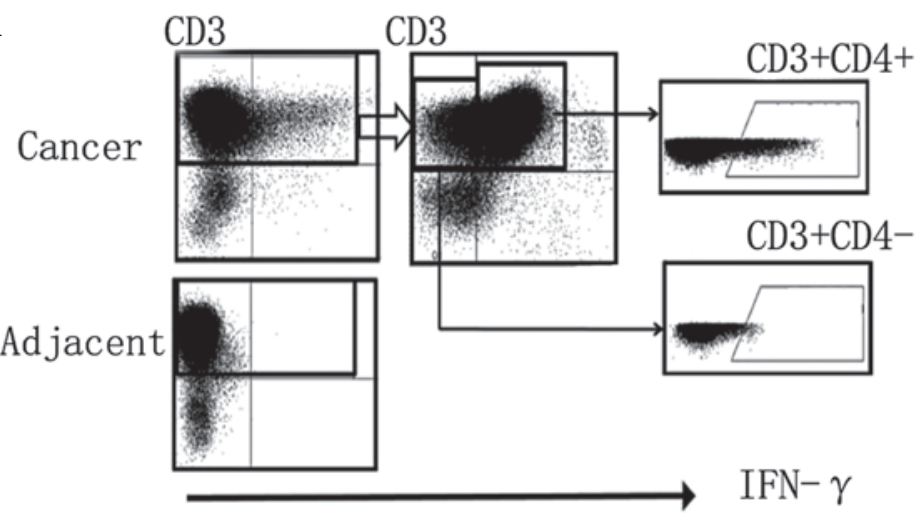

B

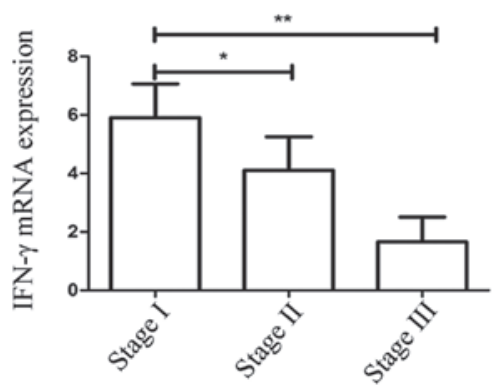

IFN- $\gamma$ mRNA

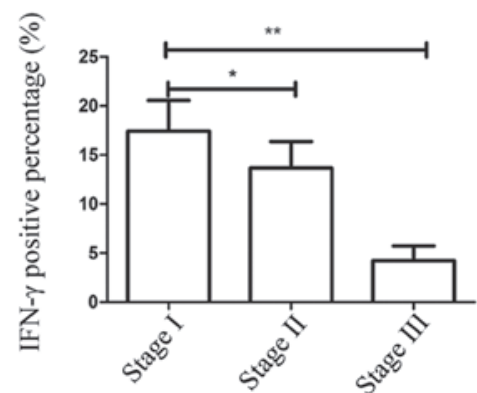

C

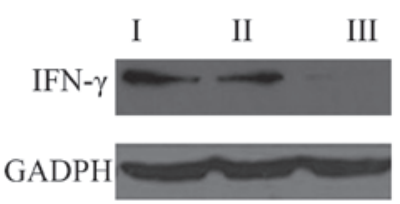

Figure 2. Expression of IFN- $\gamma$ in clinical gastric cancer tissue samples. (A) Fluorescence-activated cell sorting indicated that IFN- $\gamma$ was secreted by CD3(+) CD4(+) cells, which indicated that these were Th1 cells. (B) Expression of IFN- $\gamma$ mRNA and the percentage of IFN- $\gamma$-positive cells was significantly higher in stage I samples than those of other stages. Values are expressed as the mean \pm standard error of the mean; ${ }^{*} \mathrm{P}<0.05,{ }^{* * *} \mathrm{P}<0.01$. (C) Expression of IFN- $\gamma$ protein was significantly higher in tissues of stage I than that in other stages. mRNA, messenger RNA; IFN- $\gamma$, interferon- $\gamma$.

A LC3

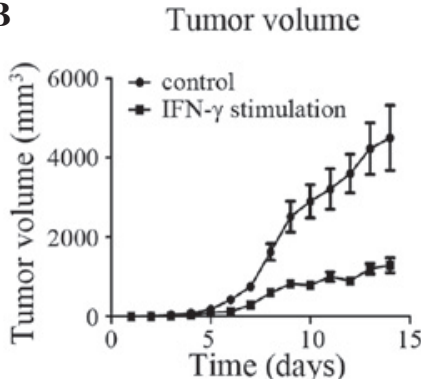

C

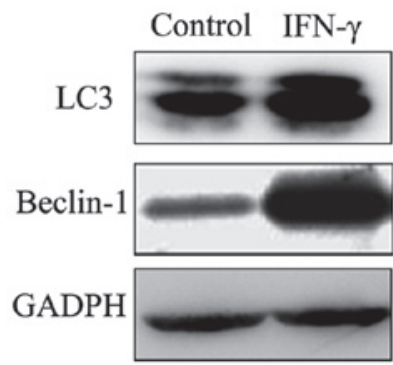

D

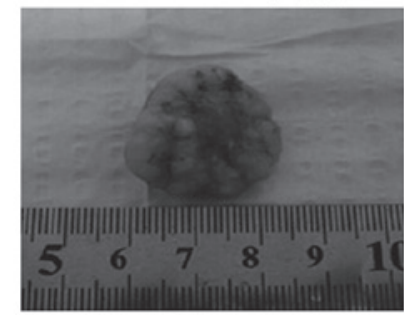

Control
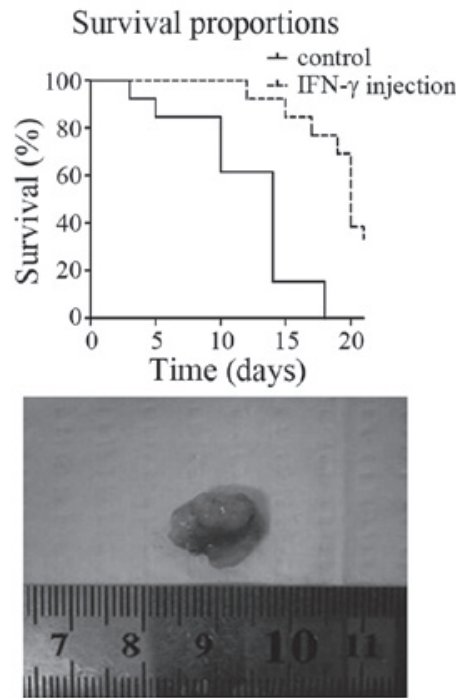

IFN- $\gamma$

Figure 3. In vitro and in vivo evaluation of the effects of IFN- $\gamma$ stimulation on tumor growth and induction of autophagy. (A) Autophagy was induced by IFN- $\gamma$ treatment of BCG-823 cells. Expression levels of autophagy-associated proteins, LC3 and Beclin-1, were significantly increased in cells stimulated with IFN- $\gamma$ compared with those treated with PBS (control). (B) Tumor volume and survival rates in a rodent xenograft model. Survival rates were significantly higher, while the mean tumor volume was significantly smaller in mice injected with IFN- $\gamma$ than those of the control mice $(\mathrm{P}<0.05$, Student's t-test). Values are expressed as the mean \pm standard error of the mean. (C) Expression levels of autophagy-associated proteins in tissues from the rodent xenograft model. Following 2 weeks of tumor growth, in vivo cancer tissues were analyzed by western blotting and it was revealed that levels of autophagy-associated proteins LC3 and Beclin-1 were increased in the IFN- $\gamma$-injection group. (D) Images of the xenograft tumor dissected from the mouse model. Xenograft growth was significantly inhibited by the injection of IFN- $\gamma$. IFN- $\gamma$, interferon- $\gamma, \mathrm{LC} 3$, microtubule-associated protein light chain 3.

Activation of autophagy inhibits tumor growth in xenograft nude mice. A rodent xenograft model was designed to detect whether autophagy could inhibit xenograft growth in vivo. The results revealed that tumor tissues from IFN- $\gamma$-treated mice exhibited significantly increased expression levels of LC3 and Beclin 1 (Fig. 3). In addition, it was noted that following 
implantation of the tumor tissues, the mice which received IFN- $\gamma$ injection had significant smaller xenograft tissues. In addition, 4 weeks later the survival rate of those mice which received IFN- $\gamma$ injection was higher than that of the control group (which only received saline). All these results indicated an anti-cancer effect of autophagy in gastric cancer cells.

\section{Discussion}

Autophagy is the process of collecting and degrading intracellular proteins and organelles in lysosomes to preserve protein and organelle quality, as well as recycling certain materials to sustain metabolism and survival during starvation (12). Autophagy is therefore important for the survival of cells and tissues, and the loss of autophagy is typically destructive (16). However, in cancer, the role of autophagy is more complex (19). Various preclinical and clinical studies have thus been undertaken, aiming to develop therapeutic agents targeting autophagy (14-20). However, compared with apoptosis, data indicating an association between cancer pathogenesis and autophagic cell death are limited $(21,22)$.

Beclin-1 is a mammalian ortholog of yeast Atg6, which has a critical role in the vesicle nucleation phase of autophagy (1). The Beclin-1 gene is located on chromosome 17q21, which is frequently deleted in breast, ovarian and prostate cancer (23). Furthermore, Beclin- $1^{+/}$mutant mice demonstrate a particularly high incidence of tumors, indicating that Beclin-1 may potentially be a haploinsufficient tumor suppressor (24-26). Thus, in the present study, the expression of Beclin-1 was evaluated in human gastric cancer specimens and cell lines. Unexpectedly, it was revealed that early-stage gastric cancer tissues expressed high levels of Beclin-1. The mRNA and protein expression levels of Beclin-1 were higher in these early-stage tissues compared with those of the adjacent normal mucosa. This result differed from the situation previously identified in breast cancer (20), indicating a distinct role for Beclin-1 in gastric cancer compared with other types of cancer. The overexpression of Beclin-1 observed in the early stages of gastric cancer in the present study may be interpreted in several ways. During the progression of cancer, cancer cells gradually acquire the capacity to evade cell death. Autophagy is an important type of cell death in tumors (23-26) and Beclin-1 may contribute to its induction. However, although in the early stages, Beclin-1 was strongly expressed in gastric cancer tissues, such changes were not observed in late-stage gastric cancer specimens (TNM stage III). By contrast, the expression levels of Beclin-1 were reduced in stage III tumors, compared with those of the adjacent mucosa. This observation indicated that Beclin-1 expression occurred at a relatively early stage. It appeared that with the progression of the tumor, gastric cancer tissues tended to lose their autophagic capacity. Notably, in the cell-based studies, similar results were observed: Expression levels of autophagy-associated proteins, Beclin-1 and LC3, were low in the poorly-differentiated BGC823 cell line but significantly higher in the well-differentiated MKN28 cell line. Further clinical analysis revealed that the depth of invasion was a significant factor affecting the expression of Beclin-1, while lymph node metastasis was not. Considering the fact that for gastric cancer, depth of invasion is more relevant in clinical diagnosis, the findings of the present study revealed that autophagy may have a significant role in this process.

Previous studies have revealed that autophagy is frequently activated in cancer cells following drug treatment $(13-16,20,24,26)$. A number of allosteric and catalytic inhibitors of mammalian target of rapamycin (mTOR), phosphoinositide-3-kinase-AKT, tyrosine kinase signaling and activators of energy sensing pathways may induce autophagy in cells (27). Therefore, it was hypothesized that autophagic cell death may be involved in the mechanism of action of certain anticancer drugs. The Akt-mTOR signaling pathway is the major negative signaling pathway against autophagy (28). Despite the fact that these chemotactic cytokines and pathways have been extensively studied, studies regarding the role of inflammation in autophagy are limited. It was previously reported that gastric carginogenesis was able to be inhibited by IFN- $\gamma$, likely through the induction of autophagy and apoptosis (29). Thus the expression and roles of IFN- $\gamma$ in gastric carcinogenesis and autophagy were evaluated in the present study. The results indicated that, notably, in the early stages of gastric cancer, the infiltration and secretion of IFN- $\gamma$ was relatively high. Activated IFN- $\gamma$-secreting cells infiltrated the cancer tissues and protein expression levels were significantly increased. However, in stage III cancer tissues, limited IFN- $\gamma$ expression was detected. It has previously been widely recognized that IFN- $\gamma$ is a significant anti-tumor cytokine (7,23-25). This change in IFN- $\gamma$ expression may occur as a result of immune evasion by the cancer cells. Since the expression of autophagy-associated protein Beclin-1 also fluctuated during cancer progression, it was hypothesized that there may be an association between these two factors.

In the present study, IFN- $\gamma$ was used to directly stimulate BGC-823 cells, which originally expressed low levels of Beclin-1. It was demonstrated that following IFN- $\gamma$ stimulation, the expression levels of LC3 and Beclin-1 were increased. As previous studies have confirmed that increasing expression of LC3 and Beclin-1 lead to increased numbers of autophagosomes (30-32), the increase in expression of these two proteins observed in the present study indicate that autophagy is activated in gastric cancer cells following in vitro stimulation with IFN- $\gamma$. Therefore, for stage III cancer cells, the lack of IFN- $\gamma$ may be a significant factor in the loss of autophagy.

The animal experiments utilized in the present study were designed to confirm the results of the in vitro experiments. To further evaluate the therapeutic effects of IFN- $\gamma$, a xenograft rodent model was generated in nude mice. The mice with xenografts typically succumbed within 2 weeks. However, following injection of IFN- $\gamma$, the survival rate of the xenograft mice significantly increased, indicating that the application of IFN- $\gamma$ may aid the limitation of tumor growth and extend the survival time. IFN- $\gamma$ has a significant role in the immune system for restricting the progression and survival of tumor cells. However, Th1 cells, the major cell which secretes IFN- $\gamma$, is typically decreased in the local area of cancer tissues or lose this ability to secrete IFN- $\gamma$. Therefore application of recombinant IFN- $\gamma$ may represent a novel therapeutic strategy for the treatment of gastric cancer. Furthermore, this effect may be mediated via the activation of autophagy.

In conclusion, the results of the current study revealed that Beclin-1, an autophagy-associated protein, exhibited a 
complex expression pattern in gastric cancer tissues at various clinical stages. In contrast to other tumors, including breast or prostate cancer, the expression of Beclin-1 in well-differentiated gastric cancer tissues and cells was higher compared with that in adjacent tissues or normal cell lines. However, in poorly-differentiated tissues and cells the opposite effect was observed. The results also revealed that there was a decrease in the infiltration and secretion of IFN- $\gamma$ associated with the progression of gastric cancer. In vitro and in vivo studies indicated that there were associations between IFN- $\gamma$ and autophagy. Additionally, IFN- $\gamma$ not only activated autophagy in gastric cancer cells, but also enhanced the survival rate in a rodent xenograft model. Thus IFN- $\gamma$ may represent a novel target for the inhibition of gastric cancer via the mechanism of autophagy.

\section{References}

1. Zhou WH, Tang F, Xu J, Wu X, Yang SB, Feng ZY, Ding YG Wan XB, Guan Z, Li HG, et al: Low expression of Beclin 1, associated with high Bcl-xL, predicts a malignant phenotype and poor prognosis of gastric cancer. Autophagy 8: 389-400, 2012.

2. Vigen RA, Kodama Y, Viset T, Fossmark R, Waldum H, Kidd M, Wang TC, Modlin IM, Chen D and Zhao CM: Immunohistochemical evidence for an impairment of autophagy in tumorigenesis of gastric carcinoids and adenocarcinomas in rodent models and patients. Histol Histopathol 28: 531-542, 2013

3. Xia P, Wang JJ, Zhao BB and Song CL: The role of beclin-1 expression in patients with gastric cancer: A meta-analysis Tumour Biol 34: 3303-3307, 2013

4. Tu SP, Quante M, Bhagat G, Takaishi S, Cui G, Yang XD, Muthuplani S, Shibata W, Fox JG, Pritchard DM and Wang TC: IFN- $\gamma$ inhibits gastric carcinogenesis by inducing epithelial cell autophagy and T-cell apoptosis. Cancer Res 71: 4247-4259, 2011

5. Vegesna AK, Miller LS, Barbe MF, Braverman AS, Khan F, and Ruggieri MR: Su1162 nicotinic receptor stimulation causes enhanced relaxation of gastric clasp, gastric sling and lower esophageal circular muscle fibers from patients with Barrett's esophagus - a possible pathophysiologic mechanism for GERD Gastroenterology 142: S440-S441, 2012.

6. Wang K, Liu R, Li J, Mao J, Lei Y, Wu J, Zeng J, Zhang T, Wu H, Chen L, et al: Quercetin induces protective autophagy in gastric cancer cells: Involvement of Akt-mTOR-and hypoxia-induced factor 1 $\alpha$-mediated signaling. Autophagy 7: 966-978, 2011.

7. Sun PH, Zhu LM, Qiao MM, Zhang YP, Jiang SH, Wu YL and Tu SP: The XAF1 tumor suppressor induces autophagic cell death via upregulation of Beclin-1 and inhibition of Akt pathway. Cancer Lett 310: 170-180, 2011.

8. Liu J, Zhang Y, Qu J, Xu L, Hou K, Zhang J, Qu X and Liu Y: $\beta$-Elemene-induced autophagy protects human gastric cancer cells from undergoing apoptosis. BMC Cancer 11: 183, 2011.

9. Kim HS, Lee SH, Do SI, Lim SJ, Park YK and Kim YW: Clinicopathologic correlation of beclin-1 expression in pancreatic ductal adenocarcinoma. Pathol Res Pract 207: 247-252, 2011

10. Rasul A, Yu B, Zhong L, Khan M, Yang H and Ma T: Cytotoxic effect of evodiamine in SGC-7901 human gastric adenocarcinoma cells via simultaneous induction of apoptosis and autophagy. Oncol Rep 27: 1481-1487, 2012.

11. Pan WR, Chen PW, Chen YL, Hsu HC, Lin CC and Chen WJ: Bovine lactoferricin B induces apoptosis of human gastric cancer cell line AGS by inhibition of autophagy at a late stage. J Dairy Sci 96: 7511-7520, 2013.

12. Park JM, Huang S, Wu TT and Sinicrope FA: 555 Association of autophagy regulator beclin 1 with response to neoadjuvant chemoradiation in rectal carcinoma. Gastroenterology 142 (Suppl 1): S111-S112, 2012

13. Washington $\mathrm{K}: 7$ th edition of the AJCC cancer staging manual: Stomach. Ann Surg Oncol 17: 3077-3079, 2010.

14. Ji D, Zhang Z, Cheng L, Chang J, Wang S, Zheng B, Zheng R, Sun Z, Wang C, Zhang Z, et al: The combination of RAD001 and MK-2206 exerts synergistic cytotoxic effects against PTEN mutant gastric cancer cells: Involvement of MAPK-dependent autophagic, but not apoptotic cell death pathway. PLoS One 9: e85116, 2014.
15. Hossain MA, Kim DH, Jang YJ, Yoon JH, Moon JO, Chung HY, Kim GY, Choi YH, Copple BL and Kim ND: Aspirin induces apoptosis in vitro and inhibits tumor growth of human hepatocellular carcinoma cells in a nude mouse xenograft model. Int J Oncol 40: 1298-1304, 2012.

16. Roesly HB, Khan MR, Chen HD, Hill KA, Narendran N, Watts GS, Chen X and Dvorak K: The decreased expression of Beclin-1 correlates with progression to esophageal adenocarcinoma: the role of deoxycholic acid. Am J Physiol Gastrointest Liver Physiol 302: G864-G872, 2012.

17. Claerhout S, Lim JY, Choi W, Park YY, Kim K, Kim SB, Lee JS, Mills GB and Cho JY: Gene expression signature analysis identifies vorinostat as a candidate therapy for gastric cancer. PLoS One 6: e24662, 2011.

18. Vigen RA, Chen D and Zhao CM: Pathobiology of gastric carcinoids and adenocarcinomas in rodent models and patients: Studies of gastrocystoplasty, gender-related factors and autophagy. In: Cell/Tissue Injury and Cytoprotection/Organoprotection in the Gastrointestinal Tract: Mechanisms, Prevention and Treatment. Filaretova LP and Takeuchi K (eds). Vol 30. S. Karger AG, Basel, pp202-211, 2012.

19. Zhou W, Yue C, Deng J, Hu R, Xu J, Feng L, Lan Q, Zhang W, $\mathrm{Ji} \mathrm{D}, \mathrm{Wu} \mathrm{J}$, et al: Autophagic protein beclin 1 serves as an independent positive prognostic biomarker for non-small cell lung cancer. PLoS One 8: e80338, 2013.

20. Won KY, Kim GY, Lim SJ and Kim YW: Decreased Beclin-1 expression is correlated with the growth of the primary tumor in patients with squamous cell carcinoma and adenocarcinoma of the lung. Hum Pathol 43: 62-68, 2012.

21. Zou Z, Wu L, Ding H, Wang Y, Zhang Y, Chen X, Chen X, Zhang CY, Zhang Q and Zen K: MicroRNA-30a sensitizes tumor cells to cis-platinum via suppressing beclin 1-mediated autophagy. J Biol Chem 287: 4148-4156, 2012.

22. Kapoor V, Paliwal D, Baskar Singh S, Mohanti BK and Das SN: Deregulation of Beclin 1 in patients with tobacco-related oral squamous cell carcinoma. Biochem Biophys Res Commun 422: 764-769, 2012.

23. Baspinar S, Bircan S, Orhan H, Kapucuoglu N and Bozkurt KK: The relation of beclin 1 and bcl-2 expressions in high grade prostatic intraepithelial neoplasia and prostate adenocarcinoma: A tissue microarray study. Pathol Res Pract 210: 412-418, 2014

24. Tong Y, You L, Liu H, Li L, Meng H, Qian Q and Qian W: Potent antitumor activity of oncolytic adenovirus expressing Beclin-1 via induction of autophagic cell death in leukemia. Oncotarget 4: 860-874, 2013.

25. Qiu DM, Wang GL, Chen L, Xu YY, He S, Cao XL, Qin J, Zhou JM, Zhang YX and E Q: The expression of beclin-1, an autophagic gene, in hepatocellular carcinoma associated with clinical pathological and prognostic significance. BMC Cancer 14: 327, 2014.

26. Sivridis E, Koukourakis MI, Mendrinos SE, Karpouzis A, Fiska A, Kouskoukis C and Giatromanolaki A: Beclin-1 and LC3A expression in cutaneous malignant melanomas: A biphasic survival pattern for beclin-1. Melanoma Res 21: 188-195, 2011.

27. Huang L, Wang S, Li SS and Yang XM: Prognostic significance of Beclin-1 expression in laryngeal squamous cell carcinoma. Pathol Oncol Res 19: 771-777, 2013.

28. Zhan Z, Li Q, Wu P, Ye Y, Tseng HY, Zhang L and Zhang XD: Autophagy-mediated HMGB1 release antagonizes apoptosis of gastric cancer cells induced by vincristine via transcriptional regulation of Mcl-1. Autophagy 8: 109-121, 2012.

29. Hasui K, Nagai T, Wang J, Jia X, Aozasa K, Izumo S, Kawano Y, Kanekura T8, Eizuru Y and Matsuyama T: Immunohistochemistry of programmed cell death in archival human pathology specimens. Cells 1: 74-88, 2012.

30. Marquez RT and Xu L: Bcl-2:Beclin 1 complex: Multiple, mechanisms regulating autophagy/apoptosis toggle switch. Am J Cancer Res 2: 214-221, 2012.

31. Wang J, Pan XL, Ding LJ, Liu DY, Da-Peng Lei and Jin T: Aberrant expression of beclin-1 and LC3 correlates with poor prognosis of human hypopharyngeal squamous cell carcinoma. PLoS One 8: e69038, 2013.

32. Hasui K, Wang J, Jia $X$, Tanaka M, Nagai T, Matsuyama $T$ and Eizuru Y: Enhanced autophagy and reduced expression of cathepsin D are related to autophagic cell death in Epstein-Barr virus-associated nasal natural killer/T-cell lymphomas: An immunohistochemical analysis of beclin-1, LC3, mitochondria (AE-1) and cathepsin D in nasopharyngeal lymphomas. Acta Histochem Cytochem 44: 119-131, 2011. 